\title{
Arquiteturas pedagógicas para simuladores de negócios: um estudo de caso com o simulador Marketplace
}

\author{
Gabriel V. Schlatter ${ }^{1}$, Patrícia A. Behar ${ }^{2}$, Eliseo B. Reategui \\ ${ }^{1}$ Escola Superior de Propaganda e Marketing - Sul \\ Porto Alegre, RS - Brazil \\ ${ }^{2}$ PGIE - Universidade Federal do Rio Grande do Sul (UFRGS) \\ Porto Alegre, RS - Brazil \\ gschlatter@espm.br, pbhar@terra.com.br, eliseoreategui@gmail.com
}

\begin{abstract}
The use of business simulations as a tool to support the development of managerial competencies is widespread in the world and rapidly expanding in Brazil. The intensive use of this feature requires a structure that supports its implementation, as well as a pedagogical basis to choose appropriate methodologies. This paper presents results of a case study with a graduate class in administration, and describes elements of pedagogical architecture defined to the simulation. We expect this could be a benchmark for future architectures, including their organizational, technological, methodological and content approaches.
\end{abstract}

Resumo. $O$ uso de simuladores de negócios como instrumento de apoio ao desenvolvimento de competências gerenciais está amplamente difundido no mundo e em rápida expansão no Brasil. $O$ uso intensivo desse recurso demanda uma estrutura que dê suporte à sua implementação, bem como uma base pedagógica que fundamente a escolha de metodologias mais adequadas. Este artigo apresenta o resultado de um estudo de caso, realizado com uma turma de pós-graduação em administração, onde se descrevem os elementos da arquitetura pedagógica definidos para um simulador de negócios. Com isto, se espera ter um referencial para futuras arquiteturas, incluindo seus aspectos organizacionais, tecnológicos, metodológicos e de conteúdos.

\section{Introdução}

As tecnologias da informação e da comunicação têm oferecido um conjunto cada vez maior de recursos para favorecer o processo de aprendizagem em todas as áreas do conhecimento. A área da administração também tem se beneficiado desses recursos para aprimorar seus métodos de ensino e aprendizagem. Um dos recursos já estabelecido no mundo [Faria, 2008] e que tem ganhado força no Brasil são os simuladores de negócios.

Simuladores de negócios têm sido tipicamente utilizados em final de curso [Bernard, 2006], uma vez que partem do pressuposto de que os fundamentos conceituais de gestão já foram oferecidos aos alunos e que, nesta etapa, sua principal necessidade passa a ser a da prática. Embora alguns cursos adotem os simuladores como complemento a uma ou outra disciplina, a maioria das escolas [Bernard, 2006] tem dedicado uma disciplina exclusiva para o uso desse recurso. 


\section{CBIE-LACLO 2015}

Anais dos Workshops do IV Congresso Brasileiro de Informática na Educação (CBIE 2015)

Entretanto, pouco se encontra na literatura sobre como esse recurso de simulação deve ser utilizado no contexto dos cursos superiores. São raras as formas estruturadas de organizar todos os componentes necessários à inclusão de simulações em programas de formação gerencial [Baldissin e outros, 2013].

Nesse contexto, surge a oportunidade de propor uma leitura das simulações sob a ótica das arquiteturas pedagógicas. Essa forma de ver o processo de ensino e aprendizagem procura caracterizar, estruturadamente, os componentes relacionados aos conteúdos, aos métodos, à organização e às tecnologias necessárias ao desenvolvimento de competências profissionais em ambientes virtuais de aprendizagem.

Assim sendo, o objetivo desse estudo é o de propor uma arquitetura pedagógica para o uso de simuladores de negócios. Isto é feito através de um estudo de caso, no qual todos os componentes do processo foram reunidos e sistematizados, definindo uma arquitetura de referência a ser adotada.

\section{Simuladores de negócios}

Simulações (ou simuladores) são softwares destinados a modelar o comportamento de algum objeto, máquina ou sistema, baseados na realidade, com a máxima fidelidade possível [Tang, 2007]. A simulação, portanto, procura reproduzir fenômenos físicos, sociais ou de negócios da forma mais próxima possível da realidade. Para tal, vale destacar a definição de Tang (2007), o qual propõe que "simuladores são sistemas de software que envolvem a simulação de experiências da vida real, destinadas ao desenvolvimento de habilidades onde os desafios apresentados reproduzem fielmente um cenário do mundo real".

Os simuladores destinados ao ensino de gestão, utilizados em cursos de administração, têm um escopo que se restringe a um ambiente de competição entre empresas, cujos gestores são os alunos. Tipicamente, numa simulação empresarial, os alunos assumem o papel de gestores de empresas que competem entre si em um mercado restrito a esses competidores. As principais informações disponibilizadas aos alunos referem-se à indústria onde deverão atuar, incluindo dados sobre o mercado e os clientes, custos de produção e desenvolvimento de tecnologias, despesas de marketing e eficiência das equipes comerciais. Na maioria dos casos, há um orçamento restrito, o qual deve ser aplicado pelos gestores nas diferentes demandas de recursos.

As decisões formalizadas pelas equipes são processadas pelo simulador, que compara as ofertas de mercado de acordo com o composto de marketing criado individualmente pelas empresas. Após o processamento, novos relatórios estão disponíveis aos gestores, repetindo-se o ciclo de decisão e análise.

Cadotte (2013) afirma que o papel do instrutor nas simulações difere muito da figura tradicional do professor, uma vez que o centro do processo de análise e decisão está com o aluno. O autor mostra que o papel do instrutor não é o de encontrar soluções para os problemas de cada time, mas sim o de levantar questões e aspectos que ainda não tenham sido contemplados nas análises prévias de cada grupo.

Sobre o encerramento, cabe registrar que, cada vez mais, dedicam-se esforços para a melhoria dessa etapa, uma vez que o processamento da simulação permite vários insights aos seus participantes. No momento em que os participantes sentam lado a lado e começam a discutir o que ocorreu ao longo da simulação, geralmente liderados pelo 


\section{CBIE-LACLO 20115}

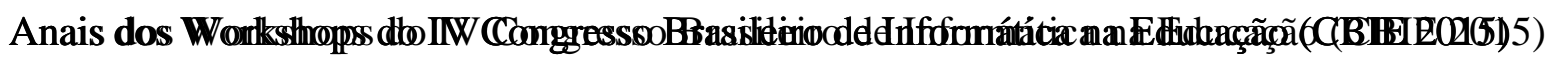

instrutor, podem surgir novos questionamentos e interpretações sobre as decisões tomadas. Um bom processamento da simulação permite aos gestores destacar os pontos importantes, assim como generalizar as lições aprendidas, de modo que os alunos possam utilizá-las em outras situações [Prensky, 2001]. Estudos comprovaram que debriefings escritos geram ainda mais ganhos de aprendizagem por forçarem os participantes a organizar a experiência da simulação sob uma ótica pessoal, questionando e reestruturando conceitos prévios [Petranek, 2000].

\section{Arquiteturas pedagógicas}

O conceito de arquitetura pedagógica surgiu como proposta de estruturação dos diversos aspectos envolvidos em formações à distância. Arquiteturas pedagógicas são estruturas que procuram sistematizar os recursos que darão suporte ao processo de aprendizagem [Carvalho et al. 2005], buscando dar autonomia ao aluno e incentivando o estudante à ação e reflexão sobre suas vivências. Behar (2009) define arquitetura pedagógica como "um sistema de premissas teóricas que representa, explica e orienta a forma como se aborda o currículo e que se concretiza nas práticas pedagógicas e nas interações professor-aluno-objeto de estudo/conhecimento". Essas diversas interações são organizadas em quatro aspectos: organizacionais, de conteúdo, metodológicos e tecnológicos [Behar, 2009].

Os aspectos organizacionais da arquitetura pedagógica dizem respeito às questões de gestão de um curso de EAD, bem como às bases legais e pedagógicas que o sustentam. Assim, se explora neste componente da arquitetura o projeto político pedagógico do curso, detalhando, em particular, o plano pedagógico no nível da disciplina e no nível institucional. Também se contempla nesse componente, a clara definição de objetivos da disciplina, a organização do tempo e do espaço e os papéis de cada um dos participantes da formação.

Os aspectos de conteúdos se caracterizam através dos componentes instrucionais, os quais podem ser qualquer tipo de material utilizado para apropriação de conhecimento [Behar, 2009]. Diz respeito ao "o quê" será trabalhado, podendo ser desde um simples material instrucional, um software educacional, páginas da web ou outros objetos de aprendizagem.

Os aspectos metodológicos, por sua vez, contemplam as atividades, as formas de interação e comunicação, os procedimentos de avaliação e a organização de todos esses elementos em uma sequência didática para a aprendizagem [Behar, 2009]. Referem-se, portanto, mais ao como será trabalhado o conteúdo, sendo definidos levando em consideração o público alvo da formação e os pressupostos epistemológicos do professor.

Por fim, os aspectos tecnológicos dizem respeito a qual ambiente virtual de aprendizagem (AVA) será utilizado, às ferramentas que darão suporte às atividades de ensino, bem como a todos os outros recursos tecnológicos disponibilizados. É importante verificar se o AVA a ser utilizado se alinha com a proposta pedagógica adotada.

\section{Estratégia metodológica}

O principal objetivo desse estudo consiste em verificar como os componentes de uma arquitetura pedagógica são implementados em atividades com simulações de negócio. 
O estudo realizado pode ser definido como uma pesquisa exploratória, segundo Malhotra (2006), a qual pode ser utilizada quando se deseja identificar variáveis que podem ser incluídas em uma pesquisa futura de caráter conclusivo.

O método de pesquisa adotado nesse trabalho foi o estudo de caso, já que o foco da análise se refere a uma simulação de negócios em particular. Segundo Yin (2001), esse método é utilizado quando se deseja responder questões do tipo "como" e "porque" sobre um contexto, bem como quando o pesquisador tem pouco controle sobre os eventos de um fenômeno contemporâneo. A unidade de estudo é uma turma de jogos de negócios de um curso de pós-graduação em administração de uma faculdade do sul do país, o qual é realizado na modalidade semipresencial. Em particular, se estudou o uso da simulação Marketplace, cujas características estão detalhadas na próxima seção.

Foi utilizada também uma pesquisa documental, a qual procurou angariar os documentos relacionados ao uso da simulação, buscando informações, tanto residentes no ambiente da simulação quanto nos materiais de apoio do curso. Utilizou-se, como técnica de coleta de dados, a observação participante, uma vez que os pesquisadores tiveram contato direto com a aplicação da simulação. Nessa participação, puderam registrar as dinâmicas de uso adotadas pelo professor, as reações dos participantes, bem como o próprio processo de avaliação final no encerramento da simulação.

Como técnica de análise de dados, se fez uma análise qualitativa básica, conforme descrita por Creswell (2010). Esta consistiu em relatar os achados da pesquisa, explicitando-os de maneira categorizada, a partir de um modelo teórico usado como referência. Particularmente, nos estudos de caso, essa descrição detalha o perfil do ambiente estudado, seguido pela análise dos dados, realizada por temas [Creswell, 2010].

\section{Análise}

Utilizando-se do referencial apresentado, se passa à descrição do caso estudado, apresentando os achados na forma de componentes da arquitetura pedagógica.

\subsection{Arquitetura pedagógica: Aspectos organizacionais}

O componente organizacional da arquitetura estudada tem origem no projeto político pedagógico da instituição e do perfil do profissional que se deseja formar. O curso na qual a disciplina é oferecida também gera diretrizes para as competências que deverão ser desenvolvidas e aprimoradas.

O caso estudado trata de um curso de pós-graduação, destinado a profissionais que desejam aprimorar suas competências gerenciais. Com esse escopo, foram propostas, como competências a serem desenvolvidas, aquelas definidas pelo Conselho Nacional de Educação para os cursos de administração de empresas [CNE, 2005]. Entre estas se destaca o pensar estrategicamente, refletir e atuar criticamente sobre a esfera da produção e exercer o processo de tomada de decisão.

O programa do curso foi definido para a disciplina do modo a que esta procurasse prover conhecimentos destinados a obter uma visão integrada do funcionamento da empresa e das diversas áreas que a compõem, tais como marketing, produção, vendas e finanças. No que diz respeito às habilidades, se espera que a disciplina desenvolva a capacidade de planejamento estratégico, implementando 


\section{CBIE-LACLO 2015}

Anais dos Workshops do IV Congresso Brasileiro de Informática na Educação (CBIE 2015)

estratégias em um ambiente competitivo e de rápida mudança. Como atitudes a serem aprimoradas, espera-se que o curso estimule o aluno a utilizar informações gerenciais de modo a diagnosticar problemas relacionados com o desempenho da empresa, embasando sua tomada de decisão.

Professores, coordenadores de curso, diretores e profissionais das diversas áreas da organização se envolvem com a disciplina desde o processo de compra de licenças de uso, programação de uma nova turma, definição dos responsáveis, suporte acadêmico e avaliação final dos alunos. Cabe à secretaria de professores a definição do momento de realização da disciplina no semestre, alocando professores para os dez encontros presenciais previstos e reservando laboratórios para a sua realização. Entretanto, o professor tem autonomia para a programação das atividades da disciplina, definindo a apresentação inicial das aulas, os dias e horários dos fechamentos das rodadas, as datas e formatos dos trabalhos complementares, os materiais de apoio e as avaliações.

Esse componente da arquitetura pedagógica é, portanto, fortemente definido pelo projeto político pedagógico da organização, servindo de base para as definições dos componentes dos outros elementos da arquitetura.

\subsection{Arquitetura pedagógica: Aspectos de conteúdos}

Nos aspectos de conteúdos da arquitetura é descrito o objeto principal de interação do aluno, que é a simulação Marketplace. Dessa forma, é descrito o ambiente simulado, as decisões a serem tomadas, a dinâmica de funcionamento da simulação e, por fim, os materiais complementares que apoiam o aluno na sua familiarização com a simulação.

A simulação Marketplace é um sistema de treinamento projetado para desenvolver a capacidade de liderança nos negócios e as habilidades necessárias à administração de uma empresa [ILS, 2013b]. A simulação estimula o pensamento estratégico e o trabalho em equipe, através de uma combinação de decisões estratégicas e desafios de mercado, inseridos em um ambiente que replica a realidade.

As equipes podem ser compostas de três a cinco alunos, os quais constroem uma empresa que visa competir com outros participantes em um mundo de negócios virtuais. São vários aspectos táticos, os quais exercitam os fundamentos de negócios, bem como a interação entre marketing, produção, logística, recursos humanos, finanças, contabilidade e gestão de equipe. Os alunos assumem o controle de uma empresa e gerenciam suas operações através de vários ciclos de decisão. Repetidamente, eles devem analisar o contexto, planejar uma estratégia para melhorá-lo, e depois executar essa estratégia com foco no futuro. Gradativamente, os alunos aprendem a adaptar sua estratégia da mesma forma com que descobrem o impacto das decisões na vida real, os conflitos gerados, os ganhos obtidos e os resultados potenciais [ILS, 2013b].

No Marketplace, o aluno gerencia uma start-up no ramo internacional de fabricação de microcomputadores. Todos os competidores iniciam a simulação exatamente com os mesmos recursos e com o mesmo conhecimento a respeito do mercado [ILS, 2013a]. Os alunos competem em 20 mercados internacionais, onde as necessidades dos clientes variam de país a país. Todos os participantes vendem seus produtos através de escritórios de vendas nos principais mercados de consumo, localizados ao redor do mundo. 


\section{CBIE-LACLO 2015}

Anais dos Workshops do IV Congresso Brasileiro de Informática na Educação (CBIE 2015)

As decisões que o aluno deverá tomar ao longo da simulação estão agrupadas em tópicos como marketing, produção e finanças [ILS, 2013a]. Todas as decisões tomadas pelos alunos têm impacto nas demonstrações financeiras da empresa, as quais podem ser apresentadas como resultado do trimestre anterior ou como projeções para o trimestre atual [ILS, 2013c].

Apesar de a simulação ser o principal meio de interação com o aluno, existe uma série de outros materiais de suporte que são disponibilizados para o mesmo, de modo tanto a facilitar o seu acompanhamento como também a reforçar o processo de aprendizagem. O principal material de suporte é um conjunto de apresentações em Power Point, nas quais estão descritos os principais passos a serem executados em cada rodada. Antes do início de cada rodada de simulação, são apresentadas informações como os pontos chaves da rodada anterior que devem ser analisados, as principais decisões a serem tomadas e, em especial, as novidades da rodada em estudo.

Além disso, são disponibilizados vários arquivos com informações complementares, como instruções e referenciais de desempenho, todos no formato de arquivos "pdf". Essas informações estão disponíveis também no próprio ambiente da simulação, numa área chamada Professor Help. Existem também artigos que dão suporte à tomada de decisão. Antes do início da sétima rodada é mostrado aos alunos um vídeo onde aparecem questões relacionadas às disputas entre grupos, num ambiente altamente competitivo. O vídeo fala do princípio das dinâmicas governantes e mostra como os grupos podem tentar buscar a melhor solução para o conjunto de empresas e não, necessariamente, a melhor solução somente para si.

Todos esses recursos são disponibilizados através do ambiente da simulação ou do AVA utilizado para apoio. Em sua maioria, os materiais complementares podem ser acessados diretamente através do AVA ou, se o aluno assim o desejar, também poderá fazer o download para impressão ou leitura prévia em sua casa.

\subsection{Arquitetura pedagógica: Aspectos metodológicos}

No que diz respeito aos aspectos metodológicos da arquitetura pedagógica, estes se compõem de uma série de escolhas sobre como o professor irá trabalhar os conteúdos, incentivando a participação dos alunos de modo a desenvolver as competências propostas pelo curso.

Para o caso estudado, há uma etapa inicial em que o professor apresenta aos alunos o que é chamado, pela instituição, de "contrato pedagógico". Neste são apresentadas as formas de funcionamento da disciplina, seus conteúdos, seu o programa, as dinâmicas de interação entre professor e aluno e a forma como este último será avaliado. O professor também descreve qual o seu papel, explicando que, numa simulação, a ele cabe o papel de um consultor. Nesse sentido, pode ser acionado sempre que os alunos tenham a necessidade de discutir algum ponto que não ficou claro, $\mathrm{e}$, principalmente, possíveis estratégias e formas de desdobramento destas em planos táticos e operacionais.

A simulação segue um ciclo que parte de uma disponibilidade de informações, as quais devem ser analisadas pelos alunos, refletindo sobre o contexto apresentado. A partir dessa reflexão, os alunos deverão propor uma estratégia competitiva para a sua organização e, como passo seguinte, exercer a tomada de decisão propriamente dita, onde as diversas táticas serão realizadas através de decisões operacionais. $\mathrm{Na}$ etapa 


\section{CBIE-LACLO 2015}

Anais dos Workshops do IV Congresso Brasileiro de Informática na Educação (CBIE 2015)

seguinte, o simulador, após receber todas as decisões dos grupos, de fato simula o comportamento do mercado, fazendo escolhas entre as diversas ofertas de produtos a partir do seu composto de marketing. Como resultado da simulação, há um novo conjunto de informações, dispostas em diferentes relatórios, os quais novamente deverão ser analisados pelos alunos, complementando o ciclo.

Nesse processo se espera que existam várias atividades de colaboração e cooperação, principalmente, fora dos encontros presenciais. A simulação utilizada possui um conjunto de registros que permite controlar o tempo que os alunos ficam logados. Relatórios disponibilizados pelo professor mostraram que, além do tempo logado durante os encontros presenciais, alguns alunos tinham mais de 3.600 minutos em conexão, isto é, mais de 60 horas de interação com a simulação. Relatos dos alunos mostram que esse tempo era referente a reuniões da equipe onde, num primeiro momento, eram feitas análises, mas, em sua maior parte, eram discutidas as alternativas estratégicas e a tomada de decisão por parte do grupo.

Sob o ponto de vista do papel do professor, conforme colocado, se espera uma intervenção pedagógica toda vez que este perceber que um determinado grupo não levou em consideração fatores essenciais num processo de análise e de tomada de decisão. O professor tem acesso a todas as decisões dos grupos antes que a rodada seja concluída e, normalmente, faz um check list, passando por cada uma das decisões. Caso algumas delas não sejam estrategicamente adequadas, incita o grupo a refletir sobre os porquês da opção adotada. Além disso, quando faz esse convite, se ocorrer do grupo não conseguir estruturar uma solução mais adequada, o professor também poderá orientá-los, através de perguntas ou de uma divisão do problema mais complexo em etapas mais simples.

Também foi encontrado na simulação estudada um recurso pedagógico que é o plano de negócios. Na quinta rodada da simulação todas as empresas são convidadas a encaminhar um plano de negócios, explicando os seus passos futuros e solicitando recursos extras, do tipo venture capital, para novos investidores. Ao grupo é dado um prazo de cinco dias para que construam um plano e enviem ao professor, o qual faz o papel de possível investidor. Posteriormente, dependendo da qualidade do plano e da consistência das propostas, o professor aloca até cinco milhões de dólares para cada um dos grupos. Em geral, os valores são diferenciados em função do detalhamento e da consistência de cada plano.

Além disso, foi solicitada uma apresentação final de cada um dos grupos, durante o último encontro presencial. Esta etapa faz parte da avaliação final, bem como de um processo de consolidação dos conhecimentos desenvolvidos durante a simulação. Cada grupo é convidado a construir uma apresentação de até dez minutos de duração, na qual dispõe sobre quais foram as suas estratégias ao longo da simulação, seus pontos fortes e fracos e, principalmente, quais foram os aprendizados. Após cada apresentação, os colegas são convidados a fazer perguntas, tirando dúvidas sobre porque foram tomadas determinadas decisões.

Finalmente, no último encontro presencial o professor também faz um apanhado geral, trazendo a sua análise dos movimentos estratégicos de cada grupo e mostrando boas decisões ou pontos fracos de cada um desses grupos. Este processo resulta numa avaliação global de toda a simulação, mostrando os momentos críticos onde o grupo 


\section{CBIE-LACLO 2015}

Anais dos Workshops do IV Congresso Brasileiro de Informática na Educação (CBIE 2015)

vencedor pode aproveitar oportunidades ou os grupos que não tiveram boas pontuações tiveram dificuldades.

A nota final de cada grupo é fortemente influenciada pela avaliação de desempenho da empresa, gerada pelo próprio simulador. A avaliação de desempenho da empresa se dá através da composição de um BSC (Balanced ScoreCard), sendo que seus principais componentes são os desempenhos financeiro e de mercado, o investimento no futuro, a geração de riqueza e a gestão de ativos [ILS, 2013 a]. Todos esses indicadores são consolidados num indicador global de BSC, o qual é calculado pelo produto dos resultados individuais. Toda vez que um indicador individual tiver um resultado negativo, seu valor é substituído por zero e o BSC global também tem seu valor zerado.

\subsection{Arquitetura pedagógica: Aspectos tecnológicos}

Como último elemento da Arquitetura Pedagógica, se faz a análise dos aspectos tecnológicos do caso estudado. A base para o processo de aprendizado é a simulação Marketplace, a qual possui sua própria plataforma, sendo residente na web. O Marketplace Business Simulation é um simulador desenvolvido nos Estados Unidos, possuindo cerca de 20.000 usuários por ano em todo o mundo. A Tectrain foi a empresa responsável pela sua implementação no Brasil, em 1998. Desde essa data, o número médio de alunos brasileiros que utilizam a simulação é de aproximadamente 2.000.

Nessa simulação, as tomadas de decisões são feitas de forma assíncrona, isto é, os grupos podem avaliar as informações e realizar suas escolhas a qualquer momento entre dois encontros presenciais. No caso em estudo, os encontros presenciais eram semanais, com quatro horas aula de duração.

Um dos momentos de sincronização das atividades consiste no fechamento da rodada, os quais se realizam na metade do período da aula presencial. Assim, esta era a data e o horário limite até o qual todos os grupos já deveriam ter suas decisões tomadas. No momento em que a rodada era fechada, o sistema processava as decisões e simulava o comportamento do mercado, gerando os novos relatórios. É neste momento (síncrono) que as apresentações da próxima rodada são feitas por parte do professor.

Como recursos tecnológicos extras, se dispõe de um e-mail individual para cada um dos integrantes e um e-mail de grupo, os quais podem ser disparados a partir da simulação. Uma forma mais elaborada de comunicação que também é oferecida é um blog, disponível para cada equipe, o qual cada participante pode editar, postar informações ou outros arquivos e se comunicar diretamente com outros elementos da sua empresa. A plataforma tecnológica da simulação possui o seu próprio help desk, o qual é composto pela equipe de suporte do representante do fornecedor da simulação no Brasil. Esta equipe tem como meta responder, em até 48 horas, qualquer consulta realizada pelos alunos integrantes da simulação.

Além da plataforma da simulação, a disciplina do simulador é integrante de um ambiente virtual de aprendizagem da própria instituição de ensino. Na instituição estudada, é utilizado o Blackboard como recurso que centraliza todos os materiais complementares. É nele, portanto, que estão todos os arquivos de instruções, as apresentações em Power Point, os artigos complementares e os vídeos. 
Caso os alunos tenham qualquer problema de conexão com um dos ambientes, a equipe de TI da instituição de ensino poderá auxiliá-los neste processo. É importante registrar que ambas as plataformas tecnológicas, quer seja a simulação quer o ambiente virtual, são acessadas pelos alunos somente mediante senhas de identificação. Isto garante que a tomada de decisão fica restrita ao grupo que realmente é responsável pela empresa em questão.

\section{Conclusão}

Como se pode ver pelo exposto, o software de simulação é apenas um dos componentes do processo de aprendizagem. Ele faz parte de uma arquitetura pedagógica, com a qual interagem vários atores (instrutor, alunos, apoiadores) e que se integra a outros recursos (equipamentos, softwares, manuais). A disponibilidade de maior capacidade computacional tem permitido o desenvolvimento de simuladores mais complexos, os quais oferecem uma interatividade com o usuário de forma mais amigável. Entretanto, seus conteúdos são cada vez mais elaborados, ensejando decisões cujo desdobramento requer análises mais profundas.

Esse aumento na complexidade do contexto de aprendizagem demanda maior reflexão, por parte do professor, sobre como se darão as diversas interações entre os participantes e a simulação. Como consequência, requer maior planejamento e compreensão sobre como ocorre a aprendizagem e sobre como cada componente da arquitetura pedagógica pode influenciar no seu desenvolvimento.

Após a conclusão da simulação, no último encontro da disciplina, cada grupo participante faz uma apresentação relatando, entre outros aspectos, quais foram os seus aprendizados. Um levantamento dos relatos dos grupos mostrou que as manifestações mais frequentes foram as de que a simulação permitiu desenvolver uma visão integrada do negócio, bem como a oportunidade de aplicar conceitos teóricos e verificar como estes funcionam na prática. Em relação à arquitetura pedagógica proposta, os alunos manifestaram que esta oportuniza a convivência com outros profissionais, enriquecendo o processo de tomada de decisão pelo compartilhar de experiências.

Além disso, a partir da forma como a vivência foi proposta, com grupos grandes de alunos em cada empresa, dois aspectos relacionados ao trabalho em equipe foram muito citados. Um deles foi o de que os participantes desenvolveram as suas próprias formas de organização do trabalho, com diferentes divisões de tarefas entre os grupos, bem como com processos de tomada de decisão muito distintos. Outro aspecto destacado pelos alunos em suas apresentações finais, foi a necessidade que tiveram de desenvolver alguma tolerância no processo de tomada de decisão, uma vez que as escolhas do grupo nem sempre eram as mesmas para todos os seus integrantes.

A proposta de sistematizar a análise do ambiente, através de uma arquitetura pedagógica, ajuda a organizar o contexto, auxiliando a tomada de decisão sobre quais recursos deverão ser utilizados. O relato de experiências como esta, pode mostrar boas práticas que venham a auxiliar novos professores na construção do seu ambiente de aprendizagem, respeitando seus pressupostos e crenças sobre como se dá a construção do conhecimento. 
CBIE-LACLO 2015

Anais dos Workshops do IV Congresso Brasileiro de Informática na Educação (CBIE 2015)

\section{Referências}

Baldissin, N. et al. (2013) Business game-based learning in management education: theory, design and successful implementation in the MEET project. Udine: The Business Game srl.

Behar, P. A. (org.). (2009) Modelos Pedagógicos para a Educação a Distância. Porto Alegre: Artmed.

Bernard, Ricardo. (2006) O método de jogos de empresa. In: Marion, José Carlos (Org.). Metodologias de ensino na área de negócios. São Paulo: Ed. Atlas.

Cadotte, Ernest R. Business Simulations: The Next Step in Management Training. (2013) Disponível em <http://www.Marketplace-simulation.com/files/docs/nextstep-in-management-training.pdf $>$. Acesso em 12/09/2013.

Carvalho, M. J.; Nevado, R. A.; Menezes, C. S. (2005) Arquitetura Pedagógicas para Educação a Distância. Concepções e Suporte Telemático. XVI Simpósio Brasileiro de Informática na Educação.

Conselho nacional de educação (CNE) / Brasil. Câmara de Educação Superior (CES). Resolução No 4 de 13 de julho de 2005. Institui as Diretrizes Curriculares Nacionais do Curso de Graduação em Administração, Bacharelado, e dá outras providências. Disponível em: <http://portal.mec.gov.br/cne/arquivos/pdf/rces004_05.pdf >. Acesso em: 01/12/2012.

Creswell, John W. (2010) Projeto de pesquisa: métodos qualitativo, quantitativo e misto. $3^{\mathrm{a}}$ ed. Porto Alegre: Artmed.

Faria, Antony J. et al. (2008) Developments in business gaming: a review of the past 40 years. Simulation \& Gaming 2009, v. 40, 22 dez. 2008.

ILS, Innovative Learning Solutions. (2013a) Business management demo. Disponível em $\quad<$ http://www.marketplace-simulation.com/flash/all-levels/demo-dynamicshortbm.html>. Acessado em $14 / 08 / 2013$.

ILS, Innovative Learning Solutions. (2013b) Educational Value of Marketplace. Disponível em <http://www.marketplace-simulation.com/college/educationalvalue.php>. Acessado em 14/08/2013.

ILS, Innovative Learning Solutions. (2013c) Instructor guidelines. Disponível em $<$ http://marketplace-simulation.com>. Acessado em 14/08/ 2013.

Malhotra, Naresh K. (2006) Pesquisa de marketing: uma orientação aplicada. Porto Alegre: Ed. Bookman.

Petranek, Charles F. (2000) Written debriefing: the next vital step in learning with simulations. Simulation \& Gaming 2000, v. 31, mar. 2000.

Prensky, Marc. (2001) "Simulations": are they games? In: Digital game-based learning. Nova York: McGraw-Hill.

Tang, Stephen et al. (2007) Describing games for learning: terms, scope and learning approaches. Game Design and Technology Workshop 2007. Proceedings. Sec. B3.

Yin, Roberto K. (2001) Estudo de caso. Porto Alegre: Ed. Bookman. 